\title{
The Impact of Magnesium Sulfate Therapy on Angiogenic Factors in Preeclampsia
}

\author{
Mary A. VADNAIS, MD, MPH ${ }^{1,2}$, Sarosh RANA, MD ${ }^{1,2}$, Hayley S. QUANT, MD ${ }^{1,2}$, Saira \\ SALAHUDDIN, MD, PhD $^{2}$, Laura E. DODGE, MPH ${ }^{2}$, Kee-Hak LIM, MD ${ }^{1,2}$, S. Ananth \\ KARUMANCHI, MD ${ }^{2,3,4}$, and Michele R. HACKER, $\mathbf{S c D}^{1,2,5}$ \\ ${ }^{1}$ Department of Obstetrics and Gynecology, Beth Israel Deaconess Medical Center and Harvard \\ Medical School, Boston, MA \\ ${ }^{2}$ Department of Obstetrics and Gynecology and Reproductive Medicine, Beth Israel Deaconess \\ Medical Center and Harvard Medical School, Boston, MA \\ ${ }^{3}$ Department of Medicine, Beth Israel Deaconess Medical Center and Harvard Medical School, \\ Boston, MA \\ ${ }^{4}$ Howard Hughes Medical Institute, Boston, MA \\ ${ }^{5}$ Department of Epidemiology, Harvard School of Public Health, Boston, MA
}

\begin{abstract}
Objective-The objective was to evaluate whether intravenous magnesium sulfate (magnesium) alters levels of angiogenic factors in women with preeclampsia.

Study Design-This was a prospective cohort study comparing women with preeclampsia treated with magnesium for seizure prophylaxis to those who were not. Serum levels of angiogenic factors, soluble fms-like tyrosine kinase 1, soluble endoglin and placental growth factor, were measured at the time of diagnosis and approximately 24 hours later. Secondary analysis compared women receiving magnesium for preeclampsia to women receiving magnesium for preterm labor. Analysis of covariance was used to compare levels at 24 hours, adjusting for levels at enrollment and potential confounders.
\end{abstract}

Results-Angiogenic factor levels did not differ between preeclampsia groups with and without magnesium or between preeclampsia and preterm labor groups treated with magnesium (all P > $0.05)$.

\footnotetext{
(C) 2011 International Society for the Study of Hypertension in Pregnancy. Published by Elsevier B.V. All rights reserved.

Corresponding author: Michele R. Hacker, ScD, Beth Israel Deaconess Medical Center, 330 Brookline Avenue, Kirstein 328, Boston, MA 02215, Business Phone: 617.667.2933, Home Phone: 857.998.1319, Fax: 617.667.5011, mhacker@bidmc.harvard.edu.

Disclosure: Dr. Karumanchi is listed as a co-inventor on multiple patents on angiogenic proteins in preeclampsia that are held by the Beth Israel Deaconess Medical Center. Dr. Karumanchi also reports having served as a consultant to Beckman Coulter and Roche Diagnostics.

The authors report no conflict of interest.

Magnesium sulfate likely decreases the risk of seizure in patients with preeclampsia by a mechanism other than altering serum levels of anti-angiogenic and pro-angiogenic factors.

Impact of magnesium sulfate on angiogenic factors

Publisher's Disclaimer: This is a PDF file of an unedited manuscript that has been accepted for publication. As a service to our customers we are providing this early version of the manuscript. The manuscript will undergo copyediting, typesetting, and review of the resulting proof before it is published in its final citable form. Please note that during the production process errorsmaybe discovered which could affect the content, and all legal disclaimers that apply to the journal pertain.
} 
Conclusion-Magnesium likely decreases seizure risk in preeclampsia by a mechanism other than altering angiogenic factor levels.

\section{Keywords}

angiogenic factors; magnesium sulfate; preeclampsia; pregnancy; toxemia

\section{Introduction}

Magnesium sulfate (magnesium) is routinely used for seizure prophylaxis in patients with preeclampsia and eclampsia. Both observational studies and randomized controlled trials suggest that administration of magnesium reduces the risk of seizures and other complications in women with preeclampsia.[1-7] The mechanism of action of magnesium is not well understood.[8,9] Possible mechanisms that have been considered include enhanced vasodilation of cerebral vasculature, production of prostacyclin which decreases vasospasm secondary to endothelial dysfunction, reduction of endothelial injury from free radicals, prevention of calcium influx into ischemic cells, a direct anticonvulsant effect via antagonism of the glutamate N-methyl-D-aspartate receptor,[10] inhibition of platelet aggregation, decrease in the release of acetylcholine at motor end plates,[11] and reduction of placental TNF-alpha secretion.[12] Other effects that have been considered and dismissed include smooth muscle relaxation, inhibition of firing of cerebral cortical neurons and reduction in local ischemia.[13]

The pathophysiology of preeclampsia is also poorly understood and may involve maternal, fetal and placental factors. An imbalance between anti-angiogenic factors, soluble fms-like tyrosine kinase 1 (sFlt1) and soluble endoglin (sEng), and pro-angiogenic factors, placental growth factor (PlGF) and vascular endothelial growth factor (VEGF), likely contributes to the development of preeclampsia.[14] Compared to women with normal pregnancies, the levels of sFlt1 and sEng are elevated in serum of women with a clinical diagnosis of preeclampsia or eclampsia, while levels of PIGF and VEGF are low.[14-17] A disturbance in angiogenic profile prior to the onset of preeclampsia $[15,18-20]$ and a correlation between levels of sFlt1 and the severity of preeclampsia have been demonstrated.[15] In addition, the over expression of sFlt1 in rats induces the preeclampsia phenotype, [14,21,22] and the use of VEGF antagonists in patients with cancer results in symptoms consistent with preeclampsia.[23,24]

A link between seizure prevention with magnesium and angiogenic factors was suggested by the report that promotion of angiogenesis by VEGF is dependent on extracellular magnesium levels.[25] We hypothesized that magnesium reduces the risk of seizure in patients with preeclampsia in part by altering serum levels of pro- and anti-angiogenic factors. Possible mechanisms for this action of magnesium include: binding of sFlt1 and sEng receptors resulting in the release of VEGF, binding of calcium channels resulting in decreased secretion of sFlt1, induction of vasodilation and decreasing placental ischemia resulting in decreased secretion of sFlt1, alteration of the translation process resulting in a decreased synthesis of sFlt1 and binding of sFlt1 resulting in decreased direct endothelial damage from sFlt1.

The objective of this study was to evaluate whether intravenous magnesium alters levels of angiogenic factors in women with preeclampsia. We measured sFlt1, sEng and PIGF at the time of diagnosis or before administration of magnesium and approximately 24 hours later. We also calculated the sFlt/PIGF ratio, which has been used as a measure of circulating angiogenic imbalance in prior studies.[18,26] 


\section{Materials and Methods}

This prospective cohort study was conducted at Beth Israel Deaconess Medical Center, Boston, MA from May 2009 through December 2010. Patients with a confirmed diagnosis of preeclampsia, as defined by American Congress of Obstetricians and Gynecologists (ACOG) criteria, and patients receiving magnesium tocolysis for preterm labor were eligible for the study.[27] Exclusion criteria included maternal age less than 18 years, gestational age less than 24 weeks and prior treatment with magnesium in the current pregnancy. The decision to treat with magnesium was made by the primary obstetrician and was not influenced by participation in this study. The protocol was approved by the Committee on Clinical Investigations at Beth Israel Deaconess Medical Center.

The primary aim of the study was to compare the levels of angiogenic factors in women diagnosed with preeclampsia who received magnesium to the levels in women with preeclampsia who did not receive magnesium. In an attempt to isolate the potential effect of magnesium on angiogenic factors, we also compared the women diagnosed with preeclampsia and treated with magnesium to women in preterm labor who received magnesium for tocolysis. A therapeutic magnesium level was defined as $\geq 4.0 \mathrm{mg} / \mathrm{dL}$.

Demographic information was collected from the participants at the time of consent. Serum samples were collected at the time of diagnosis or prior to initiating magnesium therapy (time 1) and approximately 24 hours later (time 2). All samples were collected prior to delivery. After collection, samples were centrifuged at $3000 \mathrm{RPM}$ for 10 minutes at $4^{\circ} \mathrm{C}$, aliquotted and stored at $-80^{\circ} \mathrm{C}$ until analysis. Serum sFlt1, sEng and PIGF were measured, as previously described,[14] using commercial ELISA kits (R\&D Systems, Minneapolis, $\mathrm{MN})$. All samples were run in duplicate. If more than $20 \%$ variation existed between duplicates, the assay was repeated and values were averaged. In cases where an additional ELISA was needed for one angiogenic factor, often all three factors were rerun; in these cases, the values also were averaged. Samples were randomly ordered, and a single person who was blinded to the study group performed all ELISAs.

All analyses were performed using SAS 9.2 (SAS institute Inc., Cary, NC). All tests were two sided, and $\mathrm{P}$ values $<0.05$ were considered statistically significant. Data are presented as mean ( \pm standard deviation), median (interquartile range) or proportion. The $t$, Wilcoxon, chi-square or Fisher's exact test was used for bivariate analyses based on data type and distribution.

In order to determine whether magnesium altered angiogenic factor levels in women with preeclampsia we compared levels at time 2 in the two groups while accounting for the level at time 1 , as well as potential confounders. The angiogenic factor levels were not normally distributed; thus, the values were log transformed. Analysis of covariance was used to calculate the adjusted mean log level of each of the 4 angiogenic factor or ratio values at time 2, along with 95\% confidence intervals. In each of the 4 models we included the factor level or ratio at time 1 as an independent variable, as well as baseline participant and pregnancy characteristics that were statistically significantly different between the groups and were believed to potentially affect the levels of angiogenic factors. Four additional models were created to compare women with preeclampsia treated with magnesium to women with preterm labor who were treated with magnesium.

The sample size assumptions were based on angiogenic factor data from previous research at our center.[28] We assumed a $20 \%$ decrease in sFlt1 among women with preeclampsia treated with magnesium compared with a $0 \%$ decrease in the group with preeclampsia without magnesium, a pooled standard deviation of 26 , a two-tailed $\alpha=0.05$ and $80 \%$ power. We also assumed a non-normal distribution; thus, the sample size was adjusted using an 
inflation factor of 1.15.[29] The calculation yielded a minimum required sample size of 33 women per group.

\section{Results}

A total of 37 women with preeclampsia treated with magnesium, 45 women with preeclampsia not treated with magnesium and 34 women with preterm labor treated with magnesium were enrolled and included in the analysis. With the exception of body mass index at the time of enrollment, which was higher in women treated with magnesium $(\mathrm{P}=0.048)$, there were no differences between the two groups with preeclampsia with respect to maternal characteristics such as age, race/ethnicity and gravidity (all $\mathrm{P}>0.05$; Table 1 ).

The women with preeclampsia who were treated with magnesium had greater mean blood pressure and were more likely to have severe preeclampsia, as defined by ACOG criteria, and to receive betamethasone (all $\mathrm{P}<0.05$ ), but were similar with respect to other pregnancy characteristics (Table 2).[27] Among women treated with magnesium, the women with preterm labor had a lower mean body mass index at the time of enrollment, blood pressure and gestational age at enrollment, were more likely to have a singleton gestation and were less likely to receive betamethasone (all $\mathrm{P}<0.0001$; Tables 1 and 2).

The median time between blood samples at time 1 and time 2 was 17.2 (12.6-23.2), 23.6 (19.1-24.6) and 22.6 (13.4-23.7) hours in women with preeclampsia with magnesium, preeclampsia without magnesium and in preterm labor, respectively. The time interval in the preeclampsia with magnesium group was significantly shorter than in the preeclampsia without magnesium group $(\mathrm{P}=0.007)$, but was similar in the preterm labor group $(\mathrm{P}=0.28)$. All $37(100 \%)$ of the women with preeclampsia that were treated with magnesium received a magnesium bolus of 4 grams and a maintenance dose of 2 grams per hour; the corresponding number in the preterm labor group was 31 (91.2\%). Magnesium levels from the time of the second blood draw were clinically ordered for $19(51.4 \%)$ women with preeclampsia treated with magnesium and $28(82.4 \%)$ women in the preterm labor group. Of these women, $19(100 \%)$ in the preeclampsia group and $26(92.9 \%)$ in the preterm labor group had a therapeutic level.

Among women with preeclampsia the median levels of sFlt1, sEng and the sFlt1/PIGF ratio at time 1 were similar in the group treated with magnesium compared to the group not treated with magnesium (all $\mathrm{P}>0.10$; Table 3 ). Women in preterm labor treated with magnesium had lower median levels of sFLt1 and sEng and a lower sFlt1/PIGF ratio, but higher median PlGF level at enrollment than women with preeclampsia treated with magnesium (all $\mathrm{P}<0.0001$; Table 3).

At time 2, the only difference between the two groups of women with preeclampsia was that median sEng was higher in those treated with magnesium ( $\mathrm{P}=0.03$; Table 3$)$. After adjusting for angiogenic factor level at enrollment, body mass index, systolic and diastolic blood pressure and severity of preeclampsia, the levels of angiogenic factors and the sFlt1/PIGF ratio were similar in women with preeclampsia treated with magnesium and women with preeclampsia not treated with magnesium (all $\mathrm{P}>0.50$ ). Likewise, adjusted levels did not differ between women with preeclampsia treated with magnesium and women with preterm labor treated with magnesium (all $\mathrm{P} \geq 0.17$ ). When restricting to only those women with a diagnosis of severe preeclampsia, we again saw no differences in the adjusted levels of angiogenic factors or the sFlt1/PlGF ratio at time 2 (all $\mathrm{P}>0.43$; data not presented) based on treatment with magnesium. 


\section{Discussion}

Numerous studies have demonstrated that magnesium prevents eclamptic seizures[1-7]; however, its mechanism of action is unknown.[8-9] We hypothesized that magnesium reduces seizure risk by altering pro- and anti-angiogenic factor levels. If that were its mechanism, one also would expect to observe an effect in women being treated with magnesium for conditions other than preeclampsia, such as preterm labor. We did not observe a change in angiogenic factor levels as a result of treatment with magnesium, suggesting that magnesium does not act through angiogenic factors to decrease seizure risk.

As expected, women who received magnesium were more likely to have severe preeclampsia compared to women who did not receive magnesium. This is likely due to practice patterns that result in women with severe preeclampsia more consistently receiving magnesium for seizure prophylaxis prior to delivery. Similarly, women in this group were more likely to have a higher systolic and diastolic blood pressure and more likely to receive steroids. Consistent with findings in other studies [14-18,28,30-35], sFlt1, sEng and sFlt1/ $\mathrm{PlGF}$ ratios were higher, and PIGF was lower in women with preeclampsia compared to women with preterm labor. Also anticipated, women with preterm labor were enrolled at an earlier gestational age than women with preeclampsia.

We did not find evidence to support the hypothesis that magnesium prevents seizures in women with preeclampsia by changing the angiogenic profile. It has been suggested magnesium may result in the production of prostacyclin, which decreases vasospasm secondary to endothelial dysfunction.[10] This raises the possibility of intermediates other than angiogenic factors that may influence endothelial dysfunction.

We recognize several limitations to this study. The small sample size limits our ability to do meaningful subgroup analyses; however, we were powered to detect a small difference in angiogenic factors. We did not measure concentrations of free VEGF, therefore we can not exclude the possibility that magnesium impacts levels of this angiogenic factor. Given that magnesium is used more often in severe preeclampsia, the groups differed with regards to a number of potentially important characteristics; nevertheless, we were able to account for measured potential confounders in our analysis. Magnesium levels were available for only $66 \%$ of women treated with magnesium; however, over $95 \%$ of participants treated with magnesium were exposed to a dose that was greater than a dose that has been proven to be clinically effective at seizure prevention [7] and over 95\% of magnesium levels were in the therapeutic range. Though all participants are from a single center, there is no reason to believe that this limits the generalizability of results.

This study was powered to detect a $20 \%$ change in sFlt1, which of the angiogenic factors we investigated varies the least between women with and without preeclampsia but still increases several fold in women with preeclampsia.[28] In addition to this study's power to detect a modest change in angiogenic factors, a strength of this study is the inclusion of women with a pregnancy complication other than preeclampsia who were treated with magnesium. Our hypothesis would have been supported by observing a similar association between magnesium and the angiogenic profile among women with preterm labor.

At the time this study commenced, to our knowledge there were no data on angiogenic factors in eclampsia. Recently Vaisbuch et al. reported that angiogenic profile is altered in women with eclampsia compared to women with a normal pregnancy, but comparable to women with severe preeclampsia.[28] The authors suggest that the severity of the angiogenic imbalance is not independently associated with eclampsia, which is consistent with our findings. Expert opinion suggests that the mechanism of action of magnesium in preventing seizures is likely multi-factorial.[36] Our study suggests that intravenous 
magnesium does not prevent seizures in women with preeclampsia by altering the levels of angiogenic factors.

\section{Conclusion}

Magnesium sulfate likely decreases seizure risk in preeclampsia by a mechanism other than altering angiogenic factor levels.

\section{Acknowledgments}

We thank Dongsheng Zhang in the Karumanchi laboratory for his help with the immunoassays.

This work was conducted with support from Harvard Catalyst | The Harvard Clinical and Translational Science Center (NIH Award \#UL1 RR 025758 and financial contributions from Harvard University and its affiliated academic health care centers). S.R. is supported by Harvard Diversity and Community Partnership Faculty Fellowship Award.

\section{References}

1). Evidence from the Collaborative Eclampsia Trial. Which anticonvulsant for women with eclampsia? Lancet. 1995; 345:1455. [PubMed: 7769899]

2). Duley L, Gulmezoglu AM. Magnesium sulphate versus lytic cocktail for eclampsia. Cochrane Database Syst Rev. 2001

3). Duley L, Henderson-Smart D. Magnesium sulphate versus diazepam for eclampsia. Cochrane Database Syst Rev. 2003

4). Duley L, Henderson-Smart D. Magnesium sulphate versus phenytoin for eclampsia. Cochrane Database Syst Rev. 2003

5). Lucas MJ, Leveno KJ, Cunningham FG. A comparison of magnesium sulfate with phenytoin for the prevention of eclampsia. N Engl J Med. 1995; 333:201. [PubMed: 7791836]

6). Belfort MA, Anthony J, Saade GR, Allen JC Jr, Nimodipine Study Group. A comparison of magnesium sulfate and nimodipine for the prevention of eclampsia. N Engl J Med. 2003; 348:304. [PubMed: 12540643]

7). Altman D, Carroli G, Duley L, Farrell B, Moodley J, Neilson J, Smith D, Magpie Trial Collaboration Group. Do women with pre-eclampsia, and their babies, benefit from magnesium sulphate? The Magpie Trial: a randomised placebo-controlled trial. Lancet. 2002; 359(9321): 1877. [PubMed: 12057549]

8). Sibai BM. Magnesium sulfate is the ideal anticonvulsant in preeclampsia-eclampsia. Am J Obstet Gynecol. 1990; 162:1141-5. [PubMed: 2288560]

9). Combs J. Treatment of preeclampsia and eclampsia. Clin Pharm. 1992; 11:236-45. [PubMed: 1611813]

10). Roberts JM. Magnesium for preeclampsia and eclampsia. N Engl J Med. 1995; 333:250. [PubMed: 7791843]

11). Norwitz, E.; Repke, J. [accessed February 22, 2011] Management of preeclampsia. www.uptodate.com

12). Amash A, Weintraub AY, Sheiner E, Zeadna A, Huleihel M, Holcberg G. Possible therapeutic effect of magnesium sulfate in pre-eclampsia by the down-regulation of placental tumor necrosis factor-alpha secretion. Eur Cytokine Netw. Mar; 2010 21(1):58-64. [PubMed: 20146991]

13). Greene M. Magnesium Sulfate for Preeclampsia. N Engl J Med. 2003; 348:275-276. [PubMed: 12540639]

14). Maynard SE, Min JY, Merchan J, et al. Excess placental soluble fms-like tyrosine kinase 1 (sFlt1) may contribute to endothelial dysfunction, hypertension, and proteinruria in preeclampsia. J Clin Invest. 2003; 111(5):649-58. [PubMed: 12618519]

15). Levine RJ, Maynard SE, Qian C, et al. Circulating angiogenic factors and the risk of preeclampsia. N Engl J Med. 2004; 350(7):672-83. [PubMed: 14764923] 
16). Koga K, Osuga Y, Yoshino O, et al. Elevated serum soluble vascular endothelial growth factor receptor 1 (sVEGFR-1) levels in women with preeclampsia. J Clin Endocrinol Metab. 2003; 88:2348-51. [PubMed: 12727995]

17). Tsatsaris V, Goffin F, Munaut C, et al. Overexpression of the soluble vascular endothelial growth factor in preeclamptic patients: pathophysiological consequences. J Clin Endocrinol Metab. 2003; 88:5555-63. [PubMed: 14602804]

18). Romero R, Nien JK, Espinoza J, et al. A longitudinal study of angiogenic (placental growth factor) and anti-angiogenic (soluble endoglin and soluble vascular endothelial growth factor receptor-1) factors in normal pregnancy and patients destined to develop preeclampsia and deliver a small for gestational age neonate. J Matern Fetal Neonatal Med. 2008; 21:9-23. [PubMed: 18175241]

19). Polliotti BM, Fry AG, Saller DN, Mooney RA, Cox C, Miller RK. Second-trimester maternal serum placental growth factor and vascular endothelial growth factor for predicting severe, earlyonset preeclampsia. Obstet Gynecol. 2003; 188:177-82.

20). Taylor RN, Grimwood J, Taylor RS, McMaster MT, Fisher SJ, North RA. Longitudinal serum concentrations of placental growth factor: evidence for abnormal placental angiogenesis in pathologic pregnancies. Am J Obstetr Gynecol. 2003; 188:177-82.

21). Suzuki H, Ohkuchi A, Matsubara S, et al. Effect of recombinant placental growth factor 2 on hypertension induced by full-length mouse soluble fms-like tyrosine kinase 1 adenoviral vector in pregnant mice. Hypertension. Nov; 2009 54(5):1129-35. Epub 2009 Sep 28. [PubMed: 19786649]

22). Woods AK, Hoffmann DS, Weydert CJ, et al. Adenoviral delivery of VEGF121 early in pregnancy prevents spontaneous development of preeclampsia in BPH/5 mice. Jan; 2011 57(1): 94-102. Epub 2010 Nov 15.

23). Yang JC, et al. A randomized double-blind placebo controlled trial of bevacizumab (anti- VEGF antibody) demonstrating a prolongation in time to progression in patients with metastatic renal cancer: ASCO meeting abstract. Proc. Am. Soc. Clin. Oncol. 2002; 21:A15. Abstr.

24). Kabbinavar F, Hurwitz HI, Fehrenbacher L, et al. Phase II, randomized trial comparing bevacizumab plus fluorouracil (FU)/leucovorin (LV) and FU/LV alone in patients with metastatic colorectal cancer. J Clin Oncol. 2003; 21:60-5. [PubMed: 12506171]

25). Zhonghua, Xin; Xue, Guan; Bing, Za Zhi. (Chinese Journal of Cardiovascular Disease). Evidence for a major role of Mg2+ in VEGF165-mediated angiogenesis. Mar; 2007 35(3):260-4.

26). Verlohren S, Galindo A, Schlembach D, et al. An automated method for the determination of the sFlt-1/PIGF ratio in the assessment of preeclampsia. Am J Obstet Gynecol. 2010; 202:161, e1e11. [PubMed: 19850276]

27). Diagnosis and management of preeclampsia and eclampsia. ACOG Practice Bulletin No. 33 American College of Obstetricians and Gynecologists. Obstet Gynecol. 2002; 99:159-167. [PubMed: 16175681]

28). Vaisbuch E, Whitty J, Hassan SS, et al. Circulating angiogenic and antiangiogenic factors in women with eclampsia. Am J of Obstet and Gynecol. 2011; 204:152-e1. [PubMed: 21062661]

29). Lehmann, EL. Nonparametrics: statistical methods based on ranks. Holden- Day; San Francisco: 1975. p. 76-81.

30). Chaiworapongsa T, Romero R, Espinoza J, et al. Evidence supporting a role for blockade of the vascular endothelial growth factor system in the pathophysiology of preeclampsia. Am J Obstet Gynecol. 2004; 190(6):1541. [PubMed: 15284729]

31). Venkatesha S, Toporsian M, Lam C, et al. Soluble endoglin contributes to the pathogenesis of preeclampsia. Nat Med. 2006; 12(6):642. [PubMed: 16751767]

32). McKeeman GC, Ardill JE, Caldwell CM, Hunter AJ, McClure N. Soluble vascular endothelial growth factorreceptor-1 (sFlt-1) is increased throughout gestation inpatients who have preeclampsia develop. Am J Obstet Gynecol. 2004; 191:1240-1246. [PubMed: 15507947]

33). Shibata E, Rajakumar A, Powers RW, Larkin RW, Gilmour C, Bodnar LM, Crombleholme WR, Ness RB, Roberts JM, Hubel CA. Soluble fms-like tyrosine kinase 1 is increased in preeclampsia but not in normotensive pregnancies with smallfor- gestational-age neonates: Relationship to 
circulating placental growth factor. J Clin Endocrinol Metab. 2005; 90:4895-4903. [PubMed: 15886253]

34). Staff AC, Braekke K, Harsem NK, Lyberg T, Holthe MR. Circulating concentrations of sFlt1 (soluble fms-like tyrosine kinase 1) in fetal and maternal serum during pre-eclampsia. Eur J Obstet Gynecol Reprod Biol. 2005; 122:33-39. [PubMed: 15935542]

35). Robinson CJ, Johnson DD, Chang EY, Armstrong DM, Wang W. Evaluation of placenta growth factor and soluble Fms-like tyrosine kinase 1 receptor levels in mild and severe preeclampsia. Am J Obstet Gynecol. 2006; 195:255-259. [PubMed: 16813756]

36). Euser AG, Cipolla MJ. Magnesium sulfate for the treatment of eclampsia: a brief review. Stroke. Apr; 2009 40(4):1169-75. Epub 2009 Feb 10. [PubMed: 19211496] 


\begin{tabular}{|c|c|c|c|c|c|c|c|}
\hline 2 & 晃 & $\overline{0}$ & | t) & ֻั & ః̊ & $\overrightarrow{\mathrm{m}}$ & \\
\hline 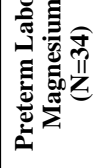 & $\begin{array}{l}\hat{n} \\
\text { +1 } \\
\dot{m} \\
\hat{m}\end{array}$ & 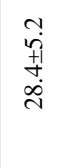 & 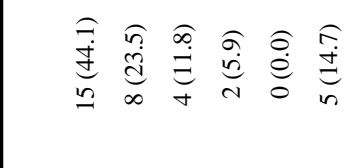 & 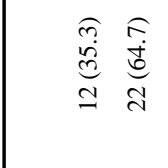 & 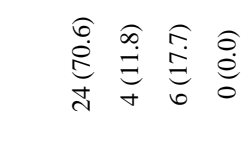 & 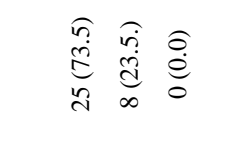 & $\stackrel{\widehat{d}}{\underline{d}}$ \\
\hline$a$ & $\stackrel{t}{\stackrel{t}{0}}$ & 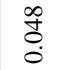 & $\stackrel{g}{0}$ & $\stackrel{f}{0}$ & $\hat{3}$ & $\hat{n}$ & \\
\hline 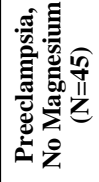 & 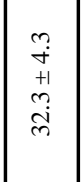 & 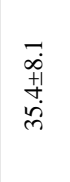 & 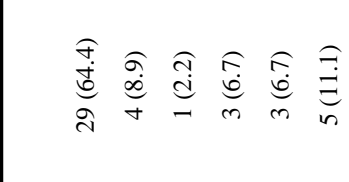 & 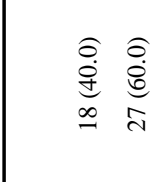 & 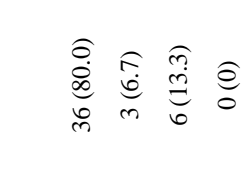 & 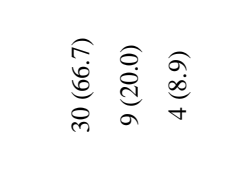 & 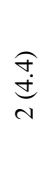 \\
\hline 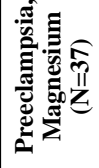 & $\begin{array}{l}m \\
\stackrel{m}{+} \\
+1 \\
\infty \\
\dot{m}\end{array}$ & 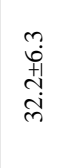 & 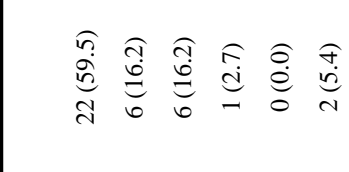 & 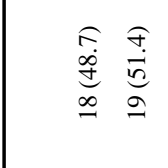 & 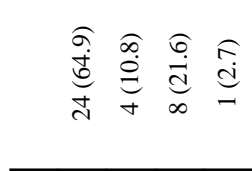 & 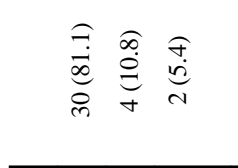 & $\underset{\mathrm{d}}{\stackrel{\widehat{d}}{-}}$ \\
\hline 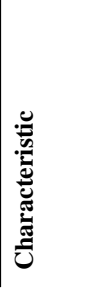 & 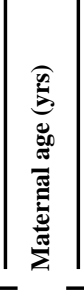 & 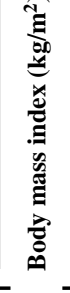 & 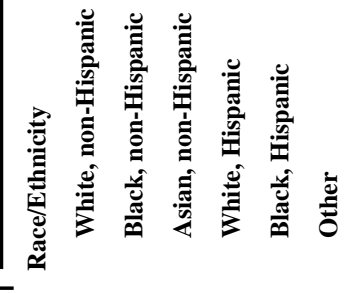 & 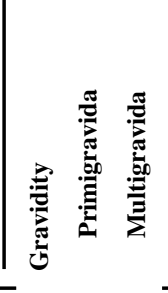 & 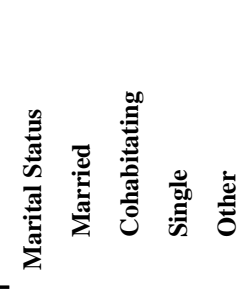 & 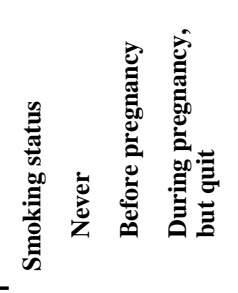 & 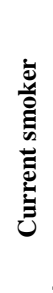 \\
\hline
\end{tabular}




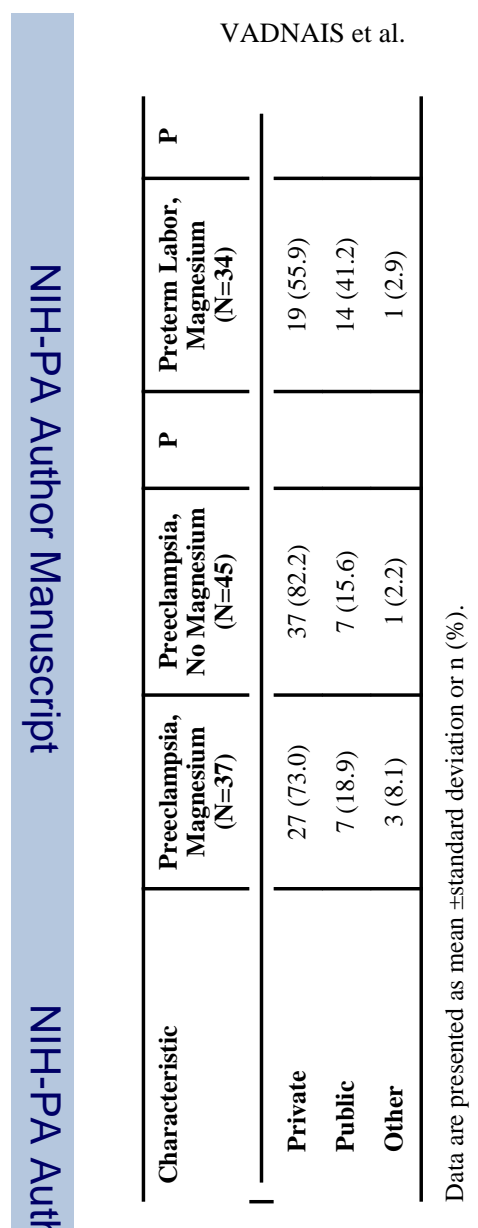

Page 10 


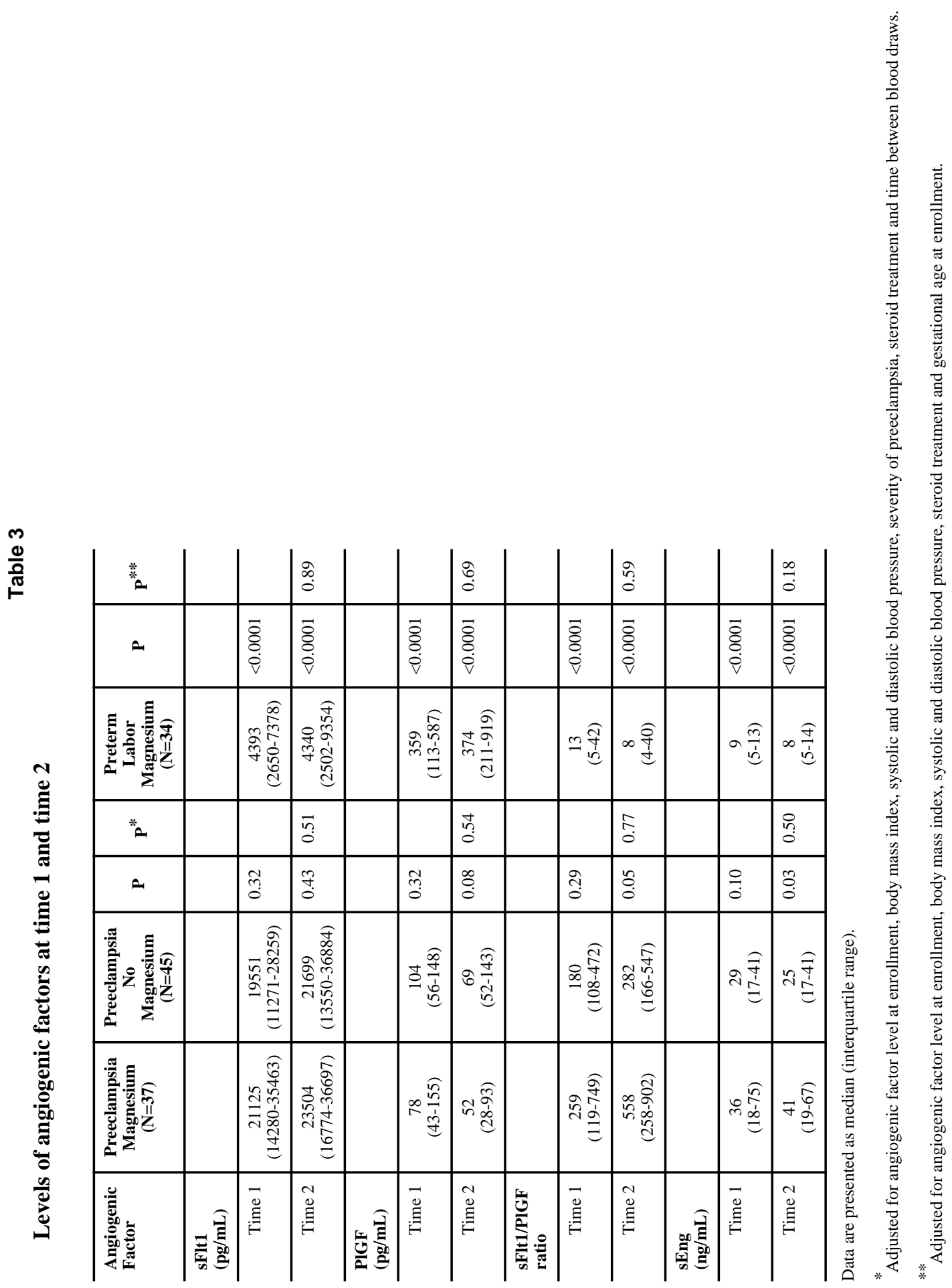

